\title{
ON THE DIMENSION OF THE PRODUCT OF TOPOLOGICAL SPACES
}

\author{
By \\ Kiiti MoRita
}

\section{Introduction.}

As in our previous paper [5], by the covering dimension of a topological space $X, \operatorname{dim} X$ in notation, we mean the least integer $n$ such that every finite normal open cover of $X$ is refined by a finite normal open cover of $X$ of order $\leqq n+1$; in case there is no such an integer $n$, we define $\operatorname{dim} X$ to be infinte. This definition was introduced for the case of Tychonoff spaces by $M$. Katětov [1] and by $\mathrm{Yu}$. Smirnov [9] independently.

In [5] we have proved the following theorem.

"Let $Y$ be a paracompact Hausdorff space which is either locally compact or $\sigma$-compact. Then $\operatorname{dim}(X \times Y) \leqq \operatorname{dim} X+\operatorname{dim} Y$ for any topological space $X$."

As is well known, those spaces which are treated conveniently in algebraic topology are $\mathrm{CW}$ complexes, and every $\mathrm{CW}$ complex is a paracompact Hausdorff space which is a countable union of locally compact closed subsets but which is neither locally compact nor $\sigma$-compact in general. Thus, the above theorem is not applicable to the case where $Y$ is a $\mathrm{CW}$ complex.

The purpose of this paper is to extend the theorem mentioned above so that it may be applied to the case with $Y$ being a $\mathrm{CW}$ complex, by establishing the following theorems.

THeOREM 1. Let $Y$ be a paracompact Hausdorff space which is a countable union of locally compact closed subspaces. Then for any topological space $X$ we have

$$
\operatorname{dim}(X \times Y) \leqq \operatorname{dim} X+\operatorname{dim} Y .
$$

Theorem 2. Let $X$ be a topological space and $Y$ a CW complex. Then

$$
\operatorname{dim}(X \times Y)=\operatorname{dim} X+\operatorname{dim} Y .
$$

TheOREM 3. Let $X$ be a topological space with $\operatorname{dim} X=1$ and $Y$ a paracompact 
Hausdorff space which is a countable union of locally compact closed subspaces. Then

$$
\operatorname{dim}(X \times Y)=\operatorname{dim} X+\operatorname{dim} Y .
$$

Hitherto these theorems have been proved for the following cases:

(a) $X$ is paracompact Hausdorff (Morita [3], Morita [4]),

(b) $Y$ is locally compact or $\sigma$-compact (Morita [5]]).

Our proof is based on the following theorem which may be of some interest when compared with the results in [6].

THEOREM 4. Let $Y$ be a topological space and $B$ a subspace of $Y$ which is locally compact, paracompact Hausdorff and is P-embedded in $Y$. Then $X \times B$ is $P$ embedded in $X \times Y$ for any topological space $X$.

Recently M. Wage [11] has proved under the Continuum Hypothesis $(\mathrm{CH})$ that the inequality

$$
\operatorname{dim}(X \times Y) \leqq \operatorname{dim} X+\operatorname{dim} Y
$$

does not hold in general even if $X \times Y$ is locally compact and normal, $\operatorname{dim} X=$ $\operatorname{dim} Y=0$, and $X=Y$.

T. Przymusiński [7] pointed out that $(\mathrm{CH})$ can be avoided by applying the methods in his paper [8] and proved also that the inequality $\left(^{*}\right)$ above does not hold in general even if $X, Y$ are Lindelöf, $X \times Y$ is normal and $\operatorname{dim} X=\operatorname{dim} Y=0$.

Thus, the assumption on $Y$ in Theorem 1 above cannot be replaced by "paracompact" or "locally compact and normal".

Theorems 1 and 2 above were announced in the author's paper: "Shape theory" (in Japanese), Sugaku, 28 (1976), 335-347.

\section{Some lemmas.}

Let us begin with Lemma 5 below.

LEMMA 5. Let $Y$ be a topological space and $B$ a subspace of $Y$ which is compact Hausdorff. If $B$ is P-embedded in $Y$, then $X \times B$ is P-embedded in $X \times Y$ for any topological space $X$.

Proof. As was shown in [5, Theorem 2.5], any normal open cover of $X \times B$ is refined by a locally finite open cover $\mathcal{G}$ of $X \times B$ of the following form:

$$
\mathcal{G}=\left\{U_{\lambda} \times V_{\lambda i} \mid 1 \leqq i \leqq r_{\lambda} ; \lambda \in \Lambda\right\}
$$

where $U=\left\{U_{\lambda} \mid \lambda \in \Lambda\right\}$ is a locally finite cozero-set cover of $X$ and $\mathcal{V}_{\lambda}=\left\{V_{\lambda i} \mid 1 \leqq i \leqq r_{\lambda}\right\}$ is a finite open cover of $B$ for each $\lambda \in \Lambda$. 
Since $B$ is P-embedded in $Y$, for each $\lambda \in \Lambda$ there is a locally finite cozero-set cover $\mathcal{H}_{\lambda}$ of $Y$ such that $\mathscr{H}_{\lambda} \cap B=\left\{H \cap B \mid H \in \mathcal{H}_{\lambda}\right\}$ refines $\mathcal{V}_{\lambda}$. The set $B$ intersects with only a finite number of the sets in $\mathcal{H}_{\lambda}$, and a locally finite union of cozerosets is also a cozero-set. Hence there is a finite cozero-set cover $\mathscr{W}_{\lambda}=\left\{W_{\lambda i} \mid 0 \leqq i \leqq r_{\lambda}\right\}$ of $Y$ such that

$$
\begin{aligned}
& W_{\lambda i} \cap B \subset V_{\lambda i} \text { for } 1 \leqq i \leqq r_{\lambda} \\
& W_{\lambda 0} \cap B=\phi
\end{aligned}
$$

Now, let us put

$$
\mathscr{M}=\left\{U_{\lambda} \times W_{\lambda i} \mid 0 \leqq i \leqq r_{\lambda} ; \lambda \in \Lambda\right\} .
$$

Then $\mathscr{M}$ is a locally finite cozero-set cover of $X \times Y$ and $\mathscr{M} \cap(X \times B)$ refines $\mathcal{G}$. This proves Lemma 5 .

Lemma 6. Let $Y$ be a Tychonoff space. Then a compact subset $B$ of $Y$ is $P$ embedded in $Y$.

Proof is obvious.

The following lemma is also easy to see.

LEMMA 7. Let $B$ be a closed subset of a normal space $Y$. If $B$ is Lindelöf, then $B$ is P-embedded in $Y$.

Therefore, if we combine Lemma 7 with Theorem 4, we have an improvement of [5, Lemma 5.6] (Lemma 7 is not used in the proof of Theorem 4) as follows.

Theorem 8. Let $Y$ be a normal Hausdorff space and $B$ a locally compact, $\sigma$ compact, closed subset of $Y$. Then $X \times B$ is P-embedded in $X \times Y$ for any topological space $X$.

\section{Proof of Theorem 4.}

Let $\left\{H_{\lambda} \mid \lambda \in \Lambda\right\}$ be a locally finite cozero-set cover of $B$ such that $C l_{B} H_{\lambda}$ (the closure in the subspace B) is compact for each $\lambda \in \Lambda$. Since $B$ is P-embedded in $Y$, there is a locally finite cozero-set cover $\mathcal{L}=\left\{L, L_{\lambda} \mid \lambda \in \Lambda\right\}$ of $Y$ such that

$$
L \cap B=\phi ; L_{\lambda} \cap B \subset H_{\lambda} \text { for } \lambda \in \Lambda \text {. }
$$

Since $\mathrm{Cl}_{B} H_{\lambda}$ is $\mathrm{P}$-embedded in $B$ and $B$ is $\mathrm{P}$-embedded in $Y, \mathrm{Cl}_{B} H_{\lambda}$ is $\mathrm{P}$-embedded in $Y$. Therefore, by Lemma $5, X \times C l_{B} H_{\lambda}$ is P-embedded in $X \times Y$.

Now, let $\mathcal{G}$ be any normal open cover of $X \times B$. Then for each $\lambda \in \Lambda$ there is a locally finite cozero-set cover $\mathcal{V}_{\lambda}$ of $X \times Y$ such that $\subset \mathcal{V}_{\lambda} \cap\left(X \times \mathrm{Cl}_{B} H_{\lambda}\right)$ refines $\mathcal{G} \cap$ $\left(X \times \mathrm{Cl}_{B} H_{\lambda}\right)$. Let us put 


$$
\mathscr{W}=\left\{X \times L, V \cap\left(X \times L_{\lambda}\right) \mid V \in \subset \mathcal{V}_{\lambda} ; \lambda \in \Lambda\right\} .
$$

Then $\mathcal{W}$ is a locally finite cozero-set cover of $X \times Y$ and $\mathscr{W} \cap(X \times B)$ refines $\mathcal{G}$. This completes the proof of Theorem 4 .

4. Proof of Theorem 1. Let $\left\{B_{i} \mid i=1,2, \cdots\right\}$ be a countable closed cover of $Y$ such that each $B_{i}$ is locally compact. Then by [5, Theorem 5.5] we have

$$
\operatorname{dim}\left(X \times B_{i}\right) \leqq \operatorname{dim} X+\operatorname{dim} B_{i}, i=1,2, \cdots .
$$

On the other hand, we have by [5, Lemma 5.8$]$

$$
\operatorname{dim} B_{i} \leqq \operatorname{dim} Y, i=1,2, \cdots .
$$

Since $X \times B_{i}$ is P-embedded in $X \times Y$ by Theorem 4 above, we get from (1), (2) and $[5$, Lemma 5.9]

$$
\operatorname{dim}(X \times Y) \leqq \operatorname{dim} X+\operatorname{dim} Y
$$

This proves Theorem 1 .

\section{Proof of Theorem 2.}

Let $B$ be a compact subset of a CW complex $Y$ which is homeomorphic to the $n$-cube $I^{n}$, where $\operatorname{dim} Y=n$. Then by Theorem 4 and [5, Lemma 5.8] we have

$$
\operatorname{dim}(X \times Y) \geqq \operatorname{dim}(X \times B) .
$$

Since $\operatorname{dim}(X \times B)=\operatorname{dim} X+\operatorname{dim} B$ by [5, Theorem 5.11] we have $\operatorname{dim}(X \times Y) \geqq \operatorname{dim} X$ $+\operatorname{dim} Y$. Now Theorem 2 follows immediately from Theorem 1 .

\section{Proof of Theorem 3 .}

Let $\left\{B_{i} \mid i=1,2, \cdots\right\}$ be a countable closed cover of $Y$ such that each $B_{i}$ is locally compact and paracompact. Then by Theorem 4 and [5, Lemmas 5.8 and 5.9] we have

$$
\operatorname{dim}(X \times Y)=\operatorname{Max}\left\{\operatorname{dim}\left(X \times B_{i}\right) \mid i=1,2, \cdots\right\} .
$$

Suppose that $\operatorname{dim}(X \times Y)=\operatorname{dim}\left(X \times B_{i}\right)$ for some $i$ and that $\operatorname{dim} Y=\operatorname{dim} B_{j}$ for some $j$. Let us put $B=B_{i} \cup B_{j}$. Then $B$ is also locally compact and paracompact and $\operatorname{dim} B=\operatorname{dim} Y, \operatorname{dim}(X \times Y)=\operatorname{dim}(X \times B)$.

On the other hand, $B$ is expressed as a disjoint union of locally compact, $\sigma$-compact closed subsets $C_{\lambda}, \lambda \in \Lambda$, of $B$. Hence we have

$$
\operatorname{dim} B=\operatorname{dim} C_{\lambda}
$$


"or some $\lambda \in \Lambda$. By [5, Theorem 5.12] we have

$$
\begin{aligned}
\operatorname{dim}(X \times Y) & =\operatorname{dim}(X \times B) \geqq \operatorname{dim}\left(X \times C_{\lambda}\right)=1+\operatorname{dim} C_{\lambda} \\
& =1+\operatorname{dim} B=1+\operatorname{dim} Y .
\end{aligned}
$$

Since $\operatorname{dim}(X \times Y) \leqq \operatorname{dim} X+\operatorname{dim} Y$ by Theorem 1, we have the desired equality $\operatorname{dim}(X \times Y)=\operatorname{dim} X+\operatorname{dim} Y$.

\section{Remarks on Theorems 4 and 8 .}

Finally we shall point out that the assumption of local compactness for $B$ is essential for the validity of Theorems 4 and 8 ; that is,

(i) Theorem 4 fails to be valid if the assumption for $B$ is weakened to " a P-embedded subspace of $Y$ which is paracompact Hausdorff and a countable union of closed locally compact subsets;

(ii) Theorem 8 fails to be valid if the assumption for $B$ is weakened to " a $\sigma$-compact closed subset of $Y$."

To prove these facts, we shall first note that the following lemma holds.

LEMMA 9. Let $C$ be a closed subset of a regular space $S$ such that the subspace $C$ and each closed subspace of $S$ contained in $S-C$ are paracompact. Then $S$ is paracompact iff $C$ is P-embedded in $S$.

Proof. The "only if" part is obvious. To prove the "if" part, suppose that $C$ is P-embedded in $S$, and let $\mathcal{G}=\left\{G_{\alpha} \mid \alpha \in \Omega\right\}$ be any open cover of $S$. Then there is a locally finite normal open cover $\left\{H_{\lambda} \mid \lambda \in \Lambda\right\}$ of $S$ such that for each $\lambda \in \Lambda$ we have

$$
H_{\lambda} \cap C \subset G_{\alpha(\lambda)} \cap C \text { for some } \alpha(\lambda) \in \Omega \text {. }
$$

Let $\Lambda_{0}=\left\{\lambda \in \Lambda \mid H_{\lambda} \cap C \neq \phi\right\}$ and put

$$
\begin{aligned}
& L_{\lambda}=H_{\lambda} \cap G_{\alpha(\lambda)} \text { for } \lambda \in \Lambda_{0}, \\
& L=\cup\left\{L_{\lambda} \mid \lambda \in \Lambda_{0}\right\} .
\end{aligned}
$$

Then $L \supset C$ and, since $S-L$ is paracompact by assumption, there is a locally finite closed cover $\left\{F_{\mu} \mid \mu \in M\right\}$ of $S-L$ which is a refinement of $\left\{G_{\alpha} \cap(S-L) \mid \alpha \in \Omega\right\}$. Then

$$
\left\{L_{\lambda}, F_{\mu} \mid \lambda \in \Lambda_{0}, \mu \in M\right\}
$$

is a locally finite cover of $S$ and it is a refinement of $\mathcal{G}$. Since $S$ is regular, this proves by virtue of a theorem of $\mathrm{E}$. Michael that $S$ is paracompact.

Now, let us return to the original subject of this section.

Let $M$ be the Michael line obtained from the Euclidean line by retopologizing it so that any subset of the set of all irrationals and the open sets in the Euclidean 
topology are open. Let $P$ be the space of all irrationals with the Euclidean topology and let us put $Q=M-P$. Then, as is proved by Michael [2], $M$ is paracompact Hausdorff and $P \times M$ is not normal. Then $P \times Q$ is not P-embedded (and hence not C-embedded) in $P \times M$ by Lemma 9 above. Let $K$ be a non-discrete compact subset of $P$. Then by [6, Theorem 1.5] $K \times P \times Q$ is not $C^{*}$-embedded in $K \times P \times M$, and by Lemma $5 \times P \times Q$ is $C^{*}$-embedded in $P \times P \times Q$. Hence $P \times P \times Q$ is not $C^{*}$. embedded in $P \times P \times M$. On the other hand, $P \times P$ is homeomorphic to $P$. Thus, $P \times Q$ is not $C^{*}$-embedded in $P \times M$, although $Q$ is $\sigma$-compact and $\mathrm{P}$-embedded in $M$.

REMARK. This example provides also a negative answer to Problem 6 of $\mathrm{M}$. Starbird [10],

\section{References}

[1] Katětov, M.: A theorem on the Lebesgue dimension. Casopis Pest. Mat. Fys., 75 (1950), 79-87.

[2] Michael, E.: The product of normal space and a metric space need not be normal. Bull. AMS., 69 (1963), 375-376.

[ 3 ] Morita, K.: On the dimension of product spaces. Amer. J. Math., 75 (1953), 205-223.

[4] Morita, K.: On the product of paracompact spaces. Proc. Japan Acad., 39 (1963), 559563.

[5] Morita, K.: Čech cohomology and covering dimension for topological spaces. Fund. Math., 87 (1975), 31-51.

[6] Morita, K. and Hoshina, T.: P-embedding and product spaces. Fund. Math., 93 (1976), 71-80.

[7] Przymusinski, T.: On the dimension of product spaces and an example of M. Wage. To appear.

[8] Przymusiński, T.: Normality and paracompactness in finite and countable cartesian products. To appear in Fund. Math.

[9] Smirnov, Yu. M.: On the dimension of proximity spaces. Mat. Sb., 38 (1956), 283-302 (in Russian).

[10] Starbird, M.: The normality of products with a compact or a metric factor. Ph. D. Thesis at Univ. Wisconsin (1974).

[11] Wage, M.: The dimension of product spaces. To appear. 